\title{
An Indoor Localization Technique Based on Ultra-Wideband AoD/AoA/ToA Estimation
}

\author{
Brecht Hanssens*, David Plets*, Emmeric Tanghe*, Claude Oestges ${ }^{\dagger}$, \\ Davy P. Gaillot ${ }^{\ddagger}$, Martine Liénard ${ }^{\ddagger}$, Luc Martens* and Wout Joseph* \\ $\ddagger$ IEMN, University of Lille 1, France, Davy.Gaillot@Univ-Lille1.fr \\ † ICTEAM, Université Catholique de Louvain, Belgium, Claude.Oestges@UCLouvain.be \\ * INTEC/WICA, Ghent University / iMinds, Belgium, Brecht.Hanssens@ INTEC.UGent.be
}

\begin{abstract}
This work presents a novel localization scheme based on Ultra-Wideband (UWB) channel sounding, employing a triangulation method that makes use of the geometrical properties of propagation paths, such as time of arrival (ToA), angle of departure (AoD) and angle of arrival (AoA). An extension to the high-resolution RiMAX algorithm was developed, facilitating the analysis of UWB frequency-dependent propagation parameters, allowing for the localization of a target node in Line-of-Sight (LoS), Obstructed-LoS (OLoS), and Non-LoS (NLoS) scenarios.
\end{abstract}

\section{INTRODUCTION}

Location information of sensor nodes in wireless sensor networks (WSNs) is crucial for applications such as target localization, tracking and guidance of mobile nodes. Many alternative approaches to the rather cost- and energy-consuming GPS can be adopted to estimate the location of the WSN's target nodes, most of which are either based on a triangulation method using angle of arrival (AoA) information, a trilateration approach using time of arrival (ToA) or received single strength indication (RSSI). However, these methods mainly focus their efforts on Line-of-Sight (LoS) scenarios and for direct propagation paths only [1].

We therefore propose a joint triangulation- and trilateration method to tackle the problem of target localization using Ultra-Wideband (UWB) channel sounding, working accurately in both LoS, Obstructed-LoS (OLoS), as well as Non-LoS (NLoS) scenarios. The main advantage of our technique is that it identifies the location of a target node using only one beacon node. Next to that, our approach can handle OLoS and NLoS scenarios exceptionally well, due to the fact that we combine the AoA with the angle of departure (AoD) information of each propagation path. In our work, we also consider the existence of multiple possible estimated target locations.

\section{MeAsurements}

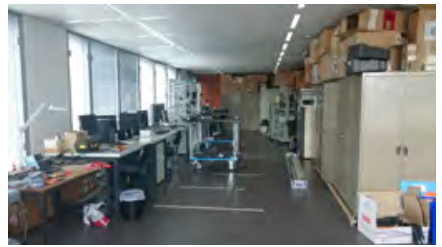

(a) Long side

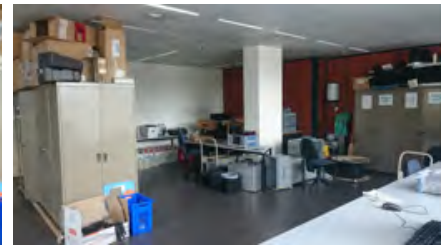

(b) Small side
Figure 1: Measurement environment.
The indoor measurements were conducted in a laboratory of Ghent University in Belgium, as depicted in Fig. 1. The base surface of the long side of the laboratory was $16 \mathrm{~m} \times 5 \mathrm{~m}$, and the small side adjacent to it was $8.5 \mathrm{~m} \times 5 \mathrm{~m}$. It was mostly equipped with metallic cabinets, tables and computers. In this indoor laboratory environment, 15 spatially distinct receiver positions (target nodes) were measured with respect to one single transmitter (beacon node). The exact locations were measured by means of a digital laser distance meter. Of these 15 positions, 8 were LoS, 4 were OLoS, and 3 were NLoS.

Wideband channel sounding measurements were carried out at each of the 15 positions with omnidirectional UWB antennas in the azimuth plane. A Vector Network Analyzer (VNA) was used to sample the complex gain of the indoor radio channel ranging from $3.1 \mathrm{GHz}$ up to $10.6 \mathrm{GHz}$ over 7501 uniformly spaced frequency points with a resolution bandwidth of $10 \mathrm{kHz}$. At both the transmit- and receiveside of the measurement system, a virtual horizontal Uniform Rectangular Array (URA) was created, dimensioned with $2 \times 2$ antennas at transmitter and receiver.

\section{Evaluation}

\section{A. UWB-RiMAX multipath estimator}

Our Multiple-Input Multiple-Output (MIMO) measurement system enables us to extract the geometrical properties of the electromagnetic waves, such as the AoD, AoA and ToA. The radio channel $\boldsymbol{h}$ can be modeled as the superposition of a deterministic part $s$ (specular multipath components (SMC)) and a stochastic part $\boldsymbol{d}$ (dense multipath components (DMC), and additive measurement noise), hence $\boldsymbol{h}=\boldsymbol{s}\left(\boldsymbol{\theta}_{\text {smc }}\right)+\boldsymbol{d}\left(\boldsymbol{\theta}_{d a n}\right)$.

The UWB-RiMAX framework, described in Algorithm 1, it is an iterative maximum-likelihood algorithm; the estimates $\hat{\boldsymbol{\theta}}_{s m c}$ and $\hat{\boldsymbol{\theta}}_{\text {dan }}$ maximize the likelihood of observing the measured frequency response $\boldsymbol{h}$ of the radio channel. The original data model of the RiMAX estimation framework follows the narrowband assumption [2], so that the reflection coefficients of materials in the environment (e.g., metallic cabinets) can no longer be considered constant with respect to frequency. In order to track these frequency-dependent propagation parameters, we developed an UWB-RiMAX framework that can process wideband measurement data. Prior to the processing, the UWB band was split up into $S=30$ sub-bands of $250 \mathrm{MHz}$ to assure frequency stationarity in each sub-band. 


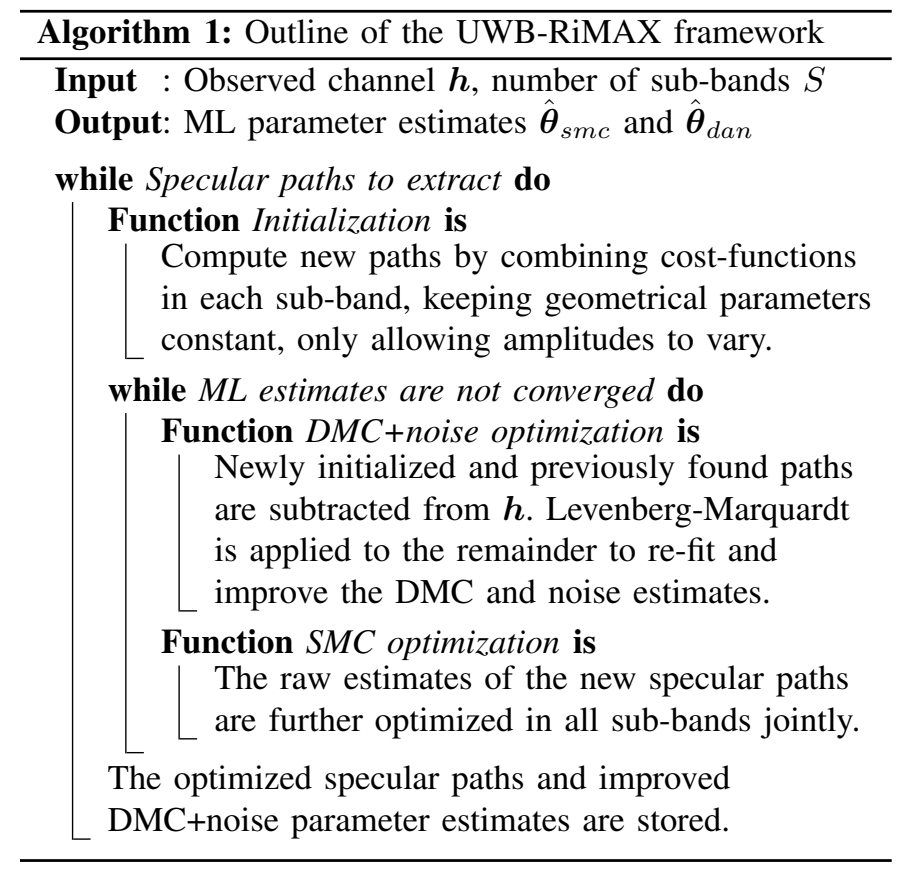

\section{B. Ray-matching algorithm}

Having access to a map with all the walls located in the surrounding area of the transmitter, this information can be used to backtrack the propagation paths between transmitter and receiver. Since we have estimates of the AoD, AoA and ToA (hence length) of the path, we can make an estimated guess about how each ray physically propagated from transmitter to where its path length equaled the ToA (assuming to be the location of the receiver). It consists of launching a ray, calculating where it impinges on a surface, and performing a reflecting- and a transmission operation (given the material is non-metallic). Finally, the ray with the computed AoA which best matches the UWB-RiMAX estimated one is considered the most-likely physical propagation path between $\mathrm{Tx}$ and $\mathrm{Rx}$.

\section{Clustering of estimations + identification of target node}

Since our UWB-RiMAX multipath framework allows us to extract several propagation paths per measurement position, we have a finite set [AoD, AoA, ToA] of geometrical parameters per estimated path. All these paths combined form a mixture of direct paths between transmitter and receiver, and multiple scattered ones. Clustering these estimated target locations with the Kim-Parks index [3] allows us to group the valid estimates, and disregard the erroneous ones. After this, we have to decide which cluster is most likely to contain the true location of the target node. We therefore established a new decision criterion, taking into account the number of propagation paths in each cluster, the amount of reflections they underwent, and their distance to the cluster-center.

\section{RESUlts}

Fig. 2 presents a map of the measurement environment with the location of the transmitter (Tx), as well as the true- and estimated receiver locations. We can see that there were no major errors in the localization of the receivers, and that the worst estimates occurred for the NLoS scenario. We can also state that our newly proposed localization technique performs very well in LoS scenarios, where as many as 7 out of the 8 measured positions achieve an accuracy below $0.70 \mathrm{~m}$. In the OLoS case, our technique still performs quite well, despite an outlier of $1.49 \mathrm{~m}$ for the $7^{\text {th }}$ position.

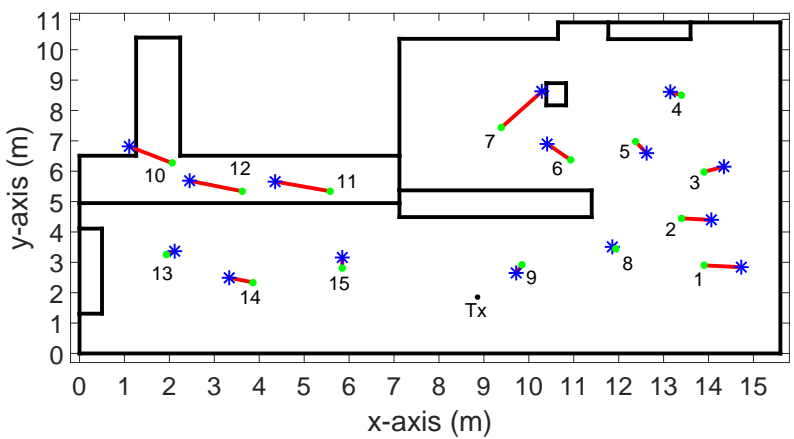

Figure 2: Location estimation of the total measurement campaign with $\mathrm{Tx}$ indicating the transmitter position. The true receiver positions are indicated with a green dot, their final estimates are indicated with a blue asterisk.

The reason why the NLoS scenario performs relatively bad might be explained by the low number of extracted paths satisfying the ray-matching criterion. Our measurements also reveal that our proposed localization technique achieves a median accuracy of $0.42 \mathrm{~m}, 0.59 \mathrm{~m}$, and $1.22 \mathrm{~m}$ in LoS, OLoS, and NLoS scenarios respectively. As expected, it is very difficult to achieve a decent accuracy in an NLoS scenario due to the electromagnetic waves having to propagate through a medium (here, plasterboard walls). However, our technique still manages to locate the receiver in the correct room.

\section{CONCLUSION}

This work presented a state-of-the-art UWB localization scheme, exploiting the geometrical properties of the estimated propagation paths. An extension to the high-resolution RiMAX algorithm was developed, after which we tested this by performing channel sounding measurements in an indoor environment. The extracted multipath parameters were subsequently used in a new Kim-Parks clustering-based localization scheme, achieving a median accuracy of $0.42 \mathrm{~m}, 0.59 \mathrm{~m}$, and $1.22 \mathrm{~m}$ in LoS, OLoS, and NLoS scenarios respectively.

\section{ACKNOWLEDGMENT}

Brecht Hanssens is funded by the Agency for Innovation by Science and Technology in Flanders (IWT). This research was supported by the project IUAP BESTCOM, "BElgian network on STochastic modelling, analysis, design and optimization of COMmunication systems".

\section{REFERENCES}

[1] L. Zwirello, T. Schipper, M. Harter, and T. Zwick. UWB Localization System for Indoor Applications: Concept, Realization and Analysis. JECE, pages 1-11, 2012.

[2] A. Richter. Estimation of Radio Channel Parameters: Models and Algorithms. PhD thesis, Technische Universität Ilmenau, Fakultät für Elektrotechnik und Informationstechnik, Ilmenau, Germany, 2005.

[3] D.-J. Kim, Y.-W. Park, and K.-J. Park. A Novel Validity Index for Determination of the Optimal Number of Clusters. IEICE Transactions on Information and Systems, 84(2):281-285, 2001. 